\title{
Shared Autoimmunity: A Case Series of 56 Patients with Immune Thrombocytopenia (ITP) Associated with Other Autoimmune Disorders
}

\author{
Lizbeth-Estefanía Díaz-Polo', Núria Pujol-Moix ${ }^{2,3^{*}}$, Blanca Jiménez ${ }^{3}$, Carme Canals ${ }^{4}$, \\ Edgardo Barranco-Charris ${ }^{1}$, Eduardo Muñiz-Díaz ${ }^{4}$, Juan-Carlos Souto² \\ ${ }^{1}$ Hematology Department, Hospital de la Santa Creu i Sant Pau, Barcelona, Spain \\ ${ }^{2}$ Hemostasis and Thrombosis Unit, Hospital de la Santa Creu i Sant Pau, Barcelona, Spain \\ ${ }^{3}$ Department of Medicine, Universitat Autònoma de Barcelona, Barcelona, Spain \\ ${ }^{4}$ Division of Immunohematology, Banc de Sang i Teixits de Catalunya, Barcelona, Spain \\ Email: *npujolmoix@gmail.com
}

Received 8 June 2016; accepted 30 July 2016; published 3 August 2016

Copyright (C) 2016 by authors and OALib.

This work is licensed under the Creative Commons Attribution International License (CC BY).

http://creativecommons.org/licenses/by/4.0/

(c) (i) Open Access

\section{Abstract}

The association of two or more autoimmune disorders in the same individual has been attributed to "shared autoimmunity" caused by the interaction between genetic and environmental factors. We present the retrospective analysis of 56 patients with Immune Thrombocytopenia (ITP) associated with other autoimmune disorders. Age, sex, clinical manifestations, platelet count, platelet autoantibodies and therapeutic responses were similar to those described in isolated ITP. However, we found a higher proportion of familial forms, suggesting a greater proportion of genetics in the etiopathogenesis of ITP associated with other autoimmune disorders. A total of 71 associated autoimmune disorders were found, the most frequent being systemic lupus erythematosus and Hashimoto thyroiditis. Most autoimmune disorders appeared before or after ITP but their simultaneous presentation was less frequent. Isolated laboratory markers of additional auto-immune disorders, mainly antinuclear and antithyroid antibodies, were found in about $20 \%$ of patients. Unlike ITP secondary to infectious or neoplastic diseases, ITP associated with other autoimmune disorders did not show significant differences with isolated ITP and it could appear indistinctly before, simultaneously, or after the associated disease. These characteristics suggest the need for a critical reconsideration of the term "secondary ITP" when ITP is associated with other autoimmune disorders.

\section{Keywords}

Shared Autoimmunity, Overlap Syndromes, Secondary Immune Thrombocytopenia,

${ }^{*}$ Corresponding author.

How to cite this paper: Díaz-Polo, L.-E., Pujol-Moix, N., Jiménez, B., Canals, C., Barranco-Charris, E., Muñiz-Díaz, E. and Souto, J.-C. (2016) Shared Autoimmunity: A Case Series of 56 Patients with Immune Thrombocytopenia (ITP) Associated with Other Autoimmune Disorders. Open Access Library Journal, 3: e2807. http://dx.doi.org/10.4236/oalib.1102807 


\title{
Systemic Lupus Erythematosus, Hashimoto Thyroiditis
}

\author{
Subject Areas: Immunology
}

\section{Introduction}

The concurrence of 2 or more Autoimmune Disorders (AD) in the same individual has been described in patients with rheumatic connective tissue diseases, autoimmune-related thyroid diseases and inflammatory bowel diseases, among others [1]. These associations have been attributed to "shared autoimmunity" due to the interaction between genetic and environmental factors [2]. Immune Thrombocytopenia (ITP) has been found to be associated with diverse AD such as Graves disease, Hashimoto thyroiditis, Systemic Lupus Erythematosus (SLE), autoimmune hemolytic anemia, and antiphospholipid syndrome [3]-[5].

ITP is a hematologic disorder characterized by thrombocytopenia due to autoimmune destruction of sensitized platelets by antibodies that react with Glycoproteins (GP) expressed on platelets and megakaryocytes including GPIIb-IIIa, GPIb-IX and GPIa-IIa [6] [7]. Bleeding, mainly mucocutaneous, is the most common clinical manifestation of ITP [6] [8]. According to the time of evolution, ITP is classified as recently diagnosed (less than 3 months), persistent (3 - 12 months) or chronic (more than 12 months) [8]. In adults, ITP presentation is usually insidious and sometimes the low platelet count might be an incidental finding [6]. ITP is also classified as primary, in the absence of other diseases, or secondary when it develops simultaneously or after other disorders that are usually autoimmune, infectious or neoplastic [8] [9]. The incidence of ITP is estimated about 2.9/100,000 person-year [10] and up to $5 \%-10 \%$ of patients are classified as having secondary ITP [7]. However, the incidence of ITP associated with other AD, but not infectious or neoplastic disorders, is not known.

The objective of the present study was to describe a case series of patients with chronic ITP associated with other AD.

\section{Methods}

We retrospectively investigated the clinical records of adult patients (older than 16 years) with chronic ITP at Hospital de la Santa Creu i Sant Pau in Barcelona, Spain. Diagnosis was based on the currently accepted international diagnostic criteria for ITP [8]. From among these patients we selected a group of 56 that also had other $\mathrm{AD}$. We collected patients' demographic and general clinical data, including assessment of bleeding and signs and symptoms of the other AD. Laboratory analyses included platelet counts, general and organ-specific autoimmune markers, and platelet serological studies using the platelet immunofluorescence test [11]. We also investigated the date of onset of the AD with respect to that of ITP as well as the presence of other family members with ITP.

The study was conducted according to the Declaration of Helsinki and it was approved by the institutional ethics committee.

\section{Results}

The mean age of the patients at initial diagnosis was 49 years (range 16 - 90), and 64\% were females (ratio female:male 1.78:1). The clinical manifestations of bleeding were generally mild, such as easy bruising and, in the majority of patients the thrombocytopenia was detected incidentally. Only 2 patients, both with very low platelet counts, had more remarkable symptoms of bleeding such as purpura and/or epistaxis. The mean platelet count at diagnosis was $67 \times 10^{9} / \mathrm{L}$ (range 10 - 100). Serologic tests were only performed in 40 patients and $26(65.0 \%)$ were positive. Of these, we found antibodies of the IgG class in 16 cases (61.6\%), of the IgG + M class in 9 (34.6\%), and of the IgG $+M+$ A class in 1 (3.8\%). Thirteen patients needed treatment due to platelet counts below $30 \times 10^{9} / \mathrm{L}$ and/or to remarkable bleeding. Nine patients had a complete response with prednisone and/or splenectomy, 3 patients reached a hemostatic response with a variety of treatments, and 1 patient was refractory to all treatments assayed. Five patients (8.9\%) had a first-degree relative with ITP, none of whom had an associated AD.

A total of $71 \mathrm{AD}$ were found, the most frequent being SLE and Hashimoto thyroiditis (Table 1). Most pa- 
tients had only 1 associated AD. However, 14 patients (25.0\%) had 2 AD besides ITP, mainly Hashimoto thyroiditis combined with SLE or with Sjogren syndrome, and 1 patient (1.8\%) had 3 associated disorders besides ITP: SLE, Hashimoto thyroiditis and thromboangiitis obliterans (Figure 1).

Most associated AD appeared after the ITP (34 disorders, 47.9\%) or before the ITP (24 disorders, 33.8\%). The least frequent situation was the simultaneous presentation of ITP and the associated AD (13 cases, 18.3\%).

We found isolated laboratory markers of additional $\mathrm{AD}$, without clinical manifestations, in 11 patients (19.6\%) (Table 2). Most of these patients had SLE or Hashimoto thyroiditis associated with ITP and the only markers found were antinuclear antibodies, antithyroid peroxidase antibodies and lupus anticoagulant.

Table 1. Autoimmune disorders associated with immune thrombocytopenia (ITP), and time of onset of them with respect to the onset of ITP.

\begin{tabular}{|c|c|c|c|c|}
\hline \multirow{2}{*}{ Autoimmune disorder } & \multirow{2}{*}{ No. (\%) } & \multicolumn{3}{|c|}{ No. according to the time of onset with respect to the onset of ITP } \\
\hline & & Before & Simultaneously & After \\
\hline Systemic lupus erythrmatosus & $20(28.2)$ & 9 & 2 & 9 \\
\hline Hashimoto thyroiditis & $11(15.5)$ & 3 & 1 & 7 \\
\hline Sjogren syndrome & $7(9.8)$ & 2 & 1 & 4 \\
\hline Psoriasis & $4(5.6)$ & 2 & 0 & 2 \\
\hline Autoimmune neutropenia & $4(5.6)$ & 1 & 3 & 0 \\
\hline Atrophic gastritis & $3(4.2)$ & 0 & 1 & 2 \\
\hline Autoimmune hemolytic anemia & $3(4.2)$ & 0 & 3 & 0 \\
\hline Ulcerative colitis & $3(4.2)$ & 0 & 0 & 3 \\
\hline Multiple sclerosis & $2(2.9)$ & 2 & 0 & 0 \\
\hline Graves disease & $2(2.9)$ & 1 & 0 & 1 \\
\hline Thromboangiitis obliterans & $2(2.9)$ & 0 & 1 & 1 \\
\hline Lichen planus & $1(1.4)$ & 0 & 0 & 1 \\
\hline Pyoderma gangernosum & $1(1.4)$ & 0 & 1 & 0 \\
\hline Primary biliary cirrhosis & $1(1.4)$ & 0 & 0 & 1 \\
\hline Celiac disease & $1(1.4)$ & 0 & 0 & 1 \\
\hline Myasthenia gravis & $1(1.4)$ & 1 & 0 & 0 \\
\hline Rheumatoid arthritis & $1(1.4)$ & 1 & 0 & 0 \\
\hline Vitiligo & $1(1.4)$ & 1 & 0 & 0 \\
\hline Antiphospholipid syndrome & $1(1.4)$ & 0 & 0 & 1 \\
\hline Autoimmune hepatitis & $1(1.4)$ & 0 & 0 & 1 \\
\hline Sarcoidosis & $1(1.4)$ & 1 & 0 & 0 \\
\hline All & $71(100.0)$ & 24 & 13 & 34 \\
\hline
\end{tabular}

Table 2. Patients with immune thrombocytopenia (ITP) and associated autoimmune disorders which also have isolated autoimmune markers (without clinical manifestations) of additional autoimmune disorders.

\begin{tabular}{|c|c|c|}
\hline Autoimmune disorders associated to ITP & No. & Isolated autoimmune markers \\
\hline Hashimoto thyroiditis & 3 & ANA \\
\hline SLE & 2 & Anti-TPO \\
\hline SLE + thromboangiits obliterans & 1 & Anti-TPO \\
\hline SLE + Hashimoto thyroiditis + thromboangiitis obliterans & 1 & Lupus anticoagulant \\
\hline Autoimmune hepatitis & 1 & ANA \\
\hline Atrophic gastritis & 1 & Anti-TPO \\
\hline Ulcerative colitis & 1 & ANA + anti-TPO \\
\hline Ulcerative colitis + vitiligo & 1 & ANA + anti-TPO \\
\hline
\end{tabular}

SLE = systemic lupus erythematosus; ANA = antinuclear antibodies; anti-TPO = antithyroid peroxidase antibodies. 


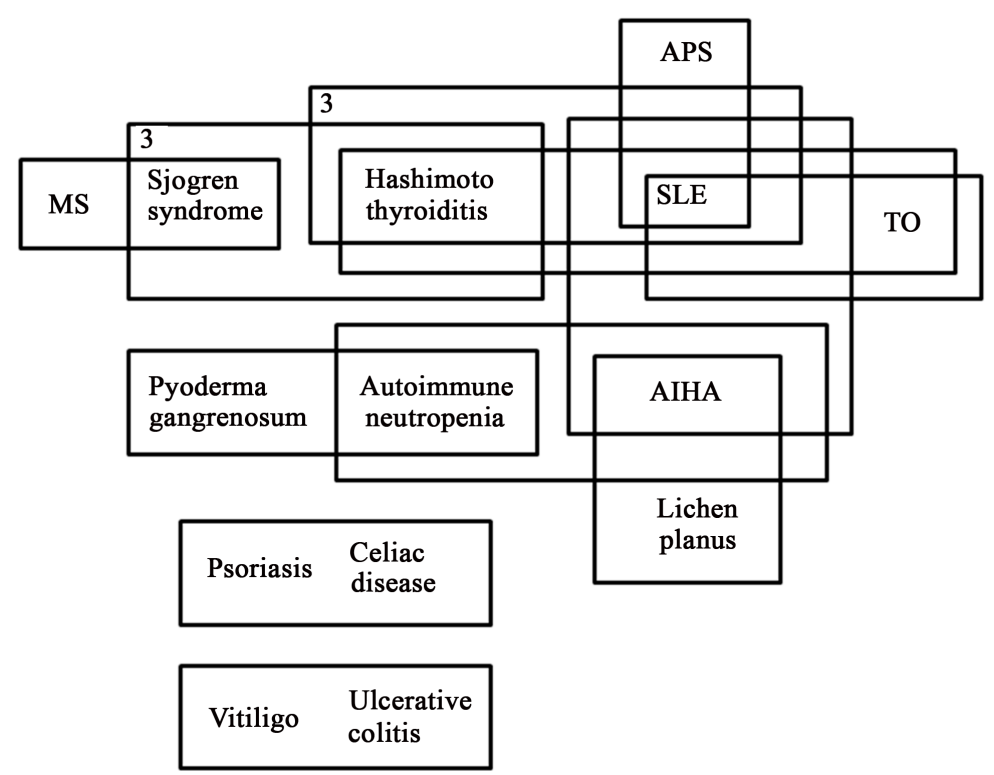

Figure 1. Graphical representation of patients with immune thrombocytopenia (ITP) and more than 1 associated autoimmune disorder. Boxes numbered " 3 " represent 3 patients with the same associations. All other boxes represent a single patient and his/her associated disorders. MS = multiple sclerosis, APS = antiphospholipid syndrome, SLE = systemic lupus erythematosus, $\mathrm{TO}=$ thromboangiitis obliterans, AIHA = autoimmune haemolytic anaemia.

\section{Discussion}

The concept of shared autoimmunity is used to refer to the development of 2 or more $\mathrm{AD}$ in the same patient or in a patient's direct relatives [12]. These associations have also been referred to as "overlap syndrome" [1] [4] and "multiple autoimmune syndrome" [2]. Some types of "autoimmune polyglandular syndrome" are also expressions of shared autoimmunity [13]. The concept of "autoimmune clustering" has also been established to describe the tendency of certain $\mathrm{AD}$ to associate with each other. Two types of associations or clusters have been described: thyrogastric or organ-specific, and lupus-associated or multisystem. Some AD, however, can be found in both clusters and others do not associate [1].

The association between ITP and other AD—such as thyroid autoimmune diseases, SLE, inflammatory bowel diseases and autoimmune hemolytic anemia—has been recognized for a long time [3] [4] [9] [14]. However, to our knowledge, no previous studies have focused on ITP and its associated AD.

We studied a case series of 56 patients with chronic ITP and diverse associated AD as examples of shared autoimmunity. The ages of the patients ranged from young adults to the very elderly, and the sex ratio was higher for women, as generally described in ITP [14]. Clinical manifestations, platelet counts and therapeutic responses did not differ significantly from other case series of adult ITP [15]-[17]. Moreover, antiplatelet antibody studies, when performed, were positive in $65 \%$ of individuals with a predominance of IgG and IgG + M class of immunoglobulins. Other studies have also reported that the IgG class is that most commonly found in antiplatelet antibodies of ITP, either alone or associated with another immunoglobulin class [18] [19]. As in other reported case series [20], we found that some patients with ITP have first-degree relatives with the same disorder. Five of our patients had a relative with ITP, none of whom had an associated AD.

Of the 71 associated $\mathrm{AD}$ found in our patients that most frequently found was SLE, either as a single associated AD or in combination with another entity (Table 1, Figure 1). The prevalence of SLE in the general population is around $0.024 \%$ but in patients with ITP it has been reported as around 3.1\% [14]. Moreover, it has been found that ITP develops in up to 1/3 of patients with SLE [9].

The second most frequently found associated AD was Hashimoto thyroiditis and, like SLE, it appeared either as a unique associated $\mathrm{AD}$ or together with another entity (Table 1, Figure 1). However, the thyroid AD Graves disease was much less frequent in our case series (Table 1). The association between thrombocytopenia and thyroid AD, including Hashimoto thyroiditis and Graves disease, has been previously described [4] [14], even in a familial context [3]. Nevertheless, specific publications about the association of ITP and Hashimoto thyroiditis 
are scarce and they are generally case reports including additional associated AD, such as Guillain-Barré syndrome [21] or rheumatoid arthritis [22].

In 7 of our patients, ITP was associated with Sjogren syndrome. Moreover, 4 of these patients presented a third AD: Hashimoto thyroiditis (3 patients) and multiple sclerosis (1 patient) (Table 1, Figure 1). The association between Sjogren syndrome and ITP has been previously described [23]. The presence of thrombocytopenia of no specified cause in Sjogren syndrome has also been described [24].

The remaining $\mathrm{AD}$ associated with ITP appeared less frequently in our patients, between 1 and 4 times (Table 1). Some of these associations have been previously described, such as those with psoriasis [25], autoimmune neutropenia [26] [27], atrophic gastritis with pernicious anemia [28], autoimmune hemolytic anemia [5] [9] [14] [26], ulcerative colitis [29], multiple sclerosis [14], lichen planus [2], pyoderma gangrenosum [27], primary biliary cirrhosis [30], celiac disease [31] [32], myastenia gravis [33], rheumatoid arthritis [34] [35], vitiligo [36], antiphospholipid syndrome [9], autoimmune hepatitis [37], and sarcoidosis [38]. However, we did not find any studies reporting the association of ITP with thromboangiitis obliterans.

Analysing the collection of associated AD found in our patients (Table 1, Figure 1), we tried to verify the situation of ITP within the established patterns of clustering in AD where it has been included in the lupusassociated cluster [1]. In our patients, the most frequently associated AD found was SLE. We also found other $\mathrm{AD}$ of the lupus-associated cluster such as Sjogren syndrome, autoimmune hemolytic anemia, rheumatoid arthritis and autoimmune hepatitis. However, our patients also had a remarkable number of thyroid AD, around 18.4\%, including Hashimoto thyroiditis and Graves disease, which belong to the thyrogastric cluster. New studies in a high number of cases are needed to further clarify this issue.

The time of onset of the associated $\mathrm{AD}$ in our patients was variable. In most patients they occurred before or after ITP and, in a minority, they occurred simultaneously with ITP (Table 1). Looking at the time of onset of specific associated disorders (we analysed only those that appeared in more than 1 patient), we observed that most of them followed the general order of presentation, that is, preferably after or before ITP. Autoimmune neutropenia and autoimmune hemolytic anemia, nevertheless, tended to occur at the same time as ITP.

Other studies in patients with multiple $\mathrm{AD}$ have also found variations in the time of onset of the various disorders in the same patient. For example, the onset of thyroid AD has been previously described [4] [16] [21] simultaneously [21] [39] and after ITP [4] [21]. Another example is ulcerative colitis, which in most cases precedes ITP but has been seen to occur after ITP in others [29]. It is of interest to emphasize the predominance of the simultaneous occurrence of autoimmune hemolytic anemia or autoimmune neutropenia with ITP, with the 3 disorders together in 1 patient. It should be taken into account that the association of 2 or 3 autoimmune cytopenias, the so-called combined immunecytopenias [26] [40] or Evans syndrome [26] [41] has been recognized for a long time but the 3 have not always occurred simultaneously [5].

The variation in the time of onset of the associated AD with respect to ITP leads us to discuss the adequacy of the term "secondary ITP", and the same could be said about other so-called "secondary autoimmune disorder" when they appear after another AD. In 2009, an international working group standardized the terminology, definitions and outcome criteria in ITP [8]. The term "secondary ITP" was proposed to broadly designate all forms of associated immunemediated thrombocytopenias, including those due to drug exposure or to an underlying disease such as SLE or HIV infection. However, not all illnesses which precede the presentation of secondary ITP are of the same nature. For example, HIV infection can cause ITP but the inverse causal relationship does not exist. Other examples include ITP secondary to hepatitis C, to drug exposure or to lymphoproliferative diseases. In contrast, from the analysis of our patients and from previously published cases, it is clear that the associated AD can appear indistinctly before, after or simultaneously with ITP, suggesting a common cause leading to both processes rather than a causal link between them. Other authors have also discussed this issue, providing views similar to those presented here [20]. Therefore, we suggest that ITP caused by virus infections, drug exposure, lymphoproliferative diseases or other causes retain the designation of "secondary ITP", and that ITP that occur associated with other AD either before, at the same time or after, be designated "associated ITP".

Eleven of our patients with ITP and 1 or more associated AD had laboratory markers of additional AD (Table 2). We found antinuclear antibodies, and/or antithyroid peroxidase antibodies in patients with diverse associated $\mathrm{AD}$ without being able to define any tendency towards a specific association between a laboratory marker and a clinical entity. Moreover, we found a lupus anticoagulant in the only patient with more than 2 associated AD. Previous studies have shown that patients with ITP frequently have isolated laboratory markers of different AD. They generally appear in chronic forms of ITP, even in children [42] [43]. Antinuclear antibodies have been 
found in adult and pediatric patients with ITP, without this meaning a risk of developing SLE [43]-[45]. Besides, a number of patients with ITP show thyroid autoantibodies without any thyroid AD [4] [31] [45]. This has also been seen in childhood forms of ITP [46]. Furthermore, the presence of a lupus anticoagulant in patients recently diagnosed with ITP has been described as a risk factor for the development of antiphospholipid syndrome [47].

Autoimmunity is a complex phenomenon where the combination of genetic and environmental factors produces a breakdown of immune tolerance. Although the trigger process is poorly understood, autoantibodies against the body's own tissues are generated, consequently producing injuries in various tissues and organs [34] [48] [49]. AD can occur in clusters or aggregations in individual patients and/or in different members of the same family. In 1997, Rose proposed that AD are unified by "shared or common threads" and he suggested adopting a unified approach to the overall AD [50]. Genetic studies carried out from this global perspective have established a genetic risk for autoimmunity based on HLA alleles and on variations in the genes that encode molecules affecting dendritic cells, B cells and T cells. Some of these genetic variations share different AD [1]. Apart from the genetic basis, AD can develop from other elements, such as female susceptibility, environmental factors and even random chance [1].

\section{Conclusion}

In conclusion, our case series of ITP associated with other AD showed no remarkable clinical or laboratory differences with the case series of isolated ITP other than a greater number of familial forms. According to shared autoimmunity theories, we can speculate that ITP patients with associated disorders and/or familial presentation have a greater proportion of genetics in their etiopathogenesis. Our patients had 1 or more associated AD and, some of them had isolated laboratory markers of additional disorders. Therefore, from a practical point of view, we recommend that the most frequent $\mathrm{AD}$ should be ruled out in all patients with ITP. This assessment should include first-degree relatives. This approach may help in the early diagnosis and treatment of the associated AD. As the onset of the associated AD differed with respect to ITP, we suggest that the expression "secondary ITP" should not be used for ITP that occurs after another AD. We propose reserving this expression for ITP caused by viral infections, drug exposure, lymphoproliferative diseases or other causes, and using the term "associated ITP" for ITP that precedes, coexists or follows other AD. We think that this nomenclature would be more consistent with the overall vision of autoimmunity.

\section{Acknowledgements}

This work was partially supported by: Instituto de Salud Carlos III of Spain (Grants PI14/00450 and RTICC RD12/0036/0071), Generalitat de Catalunya-AGAUR of Spain (Grant 2014-SGR-1281), Josep Carreras Leukaemia Research Institute and the Cellex Foundation.

\section{References}

[1] Mackay, I.R. (2009) Clustering and Commonalities among Autoimmune Diseases. Journal of Autoimmunity, 33, 170177. http://dx.doi.org/10.1016/j.jaut.2009.09.006

[2] Cojocaru, M., Cojocaru, I.M. and Silosi, I. (2010) Multiple Autoimmune Syndrome. Maedica (Buchar), 5, $132-134$.

[3] Bizzaro, N. (1992) Familial Association of Autoimmune Thrombocytopenia and Hyperthyroidism. American Journal of Hematology, 39, 294-298. http://dx.doi.org/10.1002/ajh.2830390411

[4] Cordiano, I., Betterle, C., Spadaccino, C.A., Soini, B., Girolami, A. and Fabris, F. (1998) Autoimmune Thrombocytopenia (AITP) and Thyroid Autoimmune Disease (TAD): Overlapping Syndromes? Clinical \& Experimental Immunology, 113, 373-378. http://dx.doi.org/10.1046/j.1365-2249.1998.00677.x

[5] Cines, D.B., Bussel, J.B., Liebman, H.A. and Luning Prak, E.T. (2009) The ITP Syndrome: Pathogenic and Clinical Diversity. Blood, 113, 6511-6521. http://dx.doi.org/10.1182/blood-2009-01-129155

[6] Chong, B.H. and Ho, S.J. (2005) Autoimmune Thrombocytopenia. Journal of Thrombosis and Haemostasis, 3, 17631772. http://dx.doi.org/10.1111/j.1538-7836.2005.01376.x

[7] Iraqi, M., Perdomo, J., Yan, F., Choi, P.Y.-I. and Chong, B.H. (2015) Immune Thrombocytopenia: Antiplatelet Autoantibodies Inhibit Proplatelet Formation by Megakaryocytes and Impair Platelet Production in Vitro. Haematologica, 100, 623-632. http://dx.doi.org/10.3324/haematol.2014.115634

[8] Rodeghiero, F., Stasi, R., Gernsheimer, T., Michel, M., Provan, D., Arnold, D.M., Bussel, J.B., Cines, D.B., Chong, 
B.H., Cooper, N., Godeau, B., Lechner, K., Mazzucconi, M.G., McMillan, R., Sanz, M.A., Imbach, P., Blanchette, V., Kühne, T., Ruggeri, M. and George, J.N. (2009) Standardization of Terminology, Definitions and Outcome Criteria in Immune Thrombocytopenic Purpura of Adults and Children: Report from an International Working Group. Blood, 113, 2386-2393. http://dx.doi.org/10.1182/blood-2008-07-162503

[9] Kistangari, G. and McCrae, K.R. (2013) Immune Thrombocytopenia. Hematology/Oncology Clinics of North America, 27, 495-520. http://dx.doi.org/10.1016/j.hoc.2013.03.001

[10] Moulis, G., Palmaro, A., Montastruc, J.L., Godeau, B., Lapeyre-Mestre, M. and Sailler, L. (2014) Epidemiology of Incident Immune Thrombocytopenia: A Nationwide Population-Based Study in France. Blood, 124, 3308-3315. http://dx.doi.org/10.1182/blood-2014-05-578336

[11] Von dem Borne, A.E., Hermerhorst, F.M., Van Leeuwen, E.F., Pegels, J.G., Von Riesz, E. and Engelfriet, C.P. (1980) Autoimmune Thrombocytopenia: Detection of Platelet Autoantibodies with the Suspension Immunofluorescence Test. British Journal of Haematology, 45, 319-327. http://dx.doi.org/10.1111/j.1365-2141.1980.tb07151.x

[12] Rodríguez-Reyna, T.S. and Zhernakova, A.D. (2005) Overlap Syndromes in the Context of Shared Autoimmunity. Autoimmunity, 38, 219-223. http://dx.doi.org/10.1080/08916930500050145

[13] Ergun-Longmire, B. and Maclaren, N.K. (2014) Autoimmune Polyglandular Syndromes. In: De Groot, L.J., BeckPeccoz, P., Chrousos, G., Dungan, K., Grossman, A., Hershman, J.M., Koch, C., McLachlan, R., New, M., Rebar, R., Singer, F., Vinik, A. and Weickert, M.O., Eds., Endotext [Internet], MDText.com, Inc., South Dartmouth. http://www.endotext.org

[14] Segal, J.B. (2006) Prevalence of Immune Thrombocytopenia: Analysis of Administrative Data. Journal of Thrombosis and Haemostasis, 4, 2377-2383. http://dx.doi.org/10.1111/j.1538-7836.2006.02147.x

[15] Saeidi, S., Jaseb, K., Asnafi, A.A., Rahim, F., Pourmotahari, F., Mardaniyan, S., Yousefi, H., Alghasi, A., Shahjahani, M. and Saki, N. (2014) Immune Thrombocytopenic Purpura in Children and Adults: A Comparative Retrospective Study in Iran. International Journal of Hematology-Oncology and Stem Cell Research, 8, 30-36.

[16] Aledort, L.M., Hayward, C.P., Chen, M.G., Nichol, J.L. and Bussel, J., ITP Study Group (2004) Prospective Screening of 205 Patients with ITP, Including Diagnosis, Serological Markers, and the Relationship between Platelet Counts, Endogenous Thrombosis, and Circulating Anti-Thrombopoietin Antibodies. American Journal of Hematology, 76, 205-213. http://dx.doi.org/10.1002/ajh.20104

[17] Provan, D., Stasi, R., Newland, A.C., Blanchette, V.S., Bolton-Maggs, P., Bussel, J.B., Chong, B.H., Cines, D.B., Gernsheimer, T.B., Godeau, B., Grainger, J., Greer, I., Hunt, B.J., Imbach, P.A., Lyons, G., McMillan, R., Rodeghiero, F., Sanz, M.A., Tarantino, M., Watson, S., Young, J. and Kuter, D.J. (2010) International Consensus Report on the Investigation and Management of Primary Immune Thrombocytopenia. Blood, 115, 168-186. http://dx.doi.org/10.1182/blood-2009-06-225565

[18] Chan, H., Moore, J.C., Finch, C.N., Warkentin, T.E. and Kelton, J.G. (2003) The IgG Subclasses of Platelet-Associated Autoantibodies Directed against Platelet Glycoproteins IIb/IIIa in Patients with Idiopathic Thrombocytopenic Purpura. British Journal of Haematology, 122, 818-824. http://dx.doi.org/10.1046/j.1365-2141.2003.04509.x

[19] Muñiz-Diaz, E., Pujol-Moix, N., Canals, C., Soler, J.A., Ibáñez, M., Gracia, M., Vinyet, I. and Madoz, P. (2006) The Role of Bleeding and Platelet Antibodies in Assessing the Immune Diagnosis of Primary Suspected Immune Thrombocytopenias. Vox Sanguinis, 91, 22.

[20] Kühne, T., Berchtold, W., Michaels, L.A., Wu, R., Donato, H., Espina, B., Tamary, H., Rodeghiero, F., Chitlur, M., Rischewski, J. and Imbach, P., Intercontinental Cooperative ITP Study Group (2011) Newly Diagnosed Immune Thrombocytopenia in Children and Adults: A Comparative Prospective Observational Registry of the Intercontinental Cooperative Immune Thrombocytopenia Study Group. Haematologica, 96, 1831-1837. http://dx.doi.org/10.3324/haematol.2011.050799

[21] Kohli, R.S., Bleibel, W. and Bleibel, H. (2007) Concurrent Immune Thrombocytopenic Purpura and Guillain-Barre Syndrome in a Patient with Hashimoto's Thyroiditis. American Journal of Hematology, 82, 307-308. http://dx.doi.org/10.1002/ajh.20771

[22] Yamada, Y. and Kuroe, K. (1991) A Case of Rheumatoid Arthritis Complicated with Idiopathic Thrombocytopenic Purpura and Hashimoto's Disease. Ryumachi, 31, 413-419.

[23] Jiang, B., Li, T., Guo, L., Shen, H., Ye, S. and Chen, S. (2015) Efficacy and Safety of Rituximab in Systemic Lupus Erythematosus and Sjögren Syndrome Patients With Refractory Thrombocytopenia: A Retrospective Study of 21 Cases. JCR: Journal of Clinical Rheumatology, 21, 244-250. http://dx.doi.org/10.1097/RHU.0000000000000273

[24] Ramos-Casals, M., Solans, R., Rosas, J., Camps, M.T., Gil, A., Del Pino-Montes, J., Calvo-Alen, J., Jiménez-Alonso, J., Micó, M.L., Beltrán, J., Belenguer, R. and Pallarés, L., GEMESS Study Group (2008) Primary Sjögren Syndrome in Spain: Clinical and Immunologic Expression in 1010 Patients. Medicine (Baltimore), 87, 210-219. http://dx.doi.org/10.1097/MD.0b013e318181e6af 
[25] Wu, J.J. and Nguyen, T.U. (2012) The Association of Psoriasis with Autoimmune Diseases. Journal of the American Academy of Dermatology, 67, 924-930. http://dx.doi.org/10.1016/j.jaad.2012.04.039

[26] Martino, R., Muñiz-Díaz, E., Arilla, M., Ibáñez, M., Altés, A., Guanyabens, C. and Madoz, P. (1995) Combined Autoimmune Cytopenias. Haematologica, 80, 305-310.

[27] Pujol-Moix, N., Muñiz-Diaz, E., Garcia-Patos, V., Florensa, L., Callís, M. and Gracia, M. (2006) Autoimmune Neutropenia with Cyclic Oscillations of Neutrophil Counts Associated with Cyclic Pyoderma Gangrenosum and Autoimmune Thrombocytopenia. Vox Sanguinis, 91, 6.

[28] Juncà, J., Flores, A., Granada, M.L., Jiménez, O. and Sancho, J.M. (2000) The Relationship between Idiopathic Thrombocytopenic Purpura and Pernicious Anaemia. British Journal of Haematology, 111, 513-516.

[29] Chandra, S., Finn, S. and Obah, E. (2014) Immune Thrombocytopenic Purpura in Ulcerative Colitis: A Case Report And systematic Review. Journal of Community Hospital Internal Medicine Perspectives, 4, 23386. http://dx.doi.org/10.3402/jchimp.v4.23386

[30] Toshikuni, N. and Yamato, R. (2008) Association of Primary Biliary Cirrhosis with Idiopathic Thrombocytopenic Purpura. World Journal of Gastroenterology, 14, 2451-2453. http://dx.doi.org/10.3748/wjg.14.2451

[31] Altintas, A., Pasa, S., Cil, T., Bayan, K., Gokalp, D. and Ayyildiz, O. (2008) Thyroid and Celiac Diseases Autoantibodies in Patients with Adult Chronic Idiopathic Thrombocytopenic Purpura. Platelets, 19, 252-257. http://dx.doi.org/10.1080/09537100801894651

[32] Dogan, M., Sal, E., Akbayram, S., Peker, E., Cesur, Y. and Oner, A.F. (2011) Concurrent Celiac Disease, Idiopathic Thrombocytopenic Purpura and Autoimmune Thyroiditis: A Case Report. Clinical and Applied Thrombosis/Hemostasis, 17, E13-E16. http://dx.doi.org/10.1177/1076029610378502

[33] Jansen, P.H. and Renier, W.O. (1987) Effect of Thymectomy on Myasthenia Gravis and Autoimmune Thrombocytopenic Purpura in a 13-Year-Old Girl. European Journal of Pediatrics, 146, 587-589. http://dx.doi.org/10.1007/BF02467359

[34] Johnsen, J. (2012) Pathogenesis in Immune Thrombocytopenia: New Insights. Hematology American Society of Hematology Education Program, 2012, 306-312.

[35] Horino, T. and Sasaoka, A. (2005) Immune Thrombocytopenic Purpura Associated with Rheumatoid Arthritis: Case Report. Clinical Rheumatology, 24, 641-644. http://dx.doi.org/10.1007/s10067-005-1113-x

[36] Gidwani, P., Segal, E., Shanske, A. and Driscoll, C. (2007) Chorea Associated with Antiphospholipid Antibodies in a Patient with Kabuki Syndrome. American Journal of Medical Genetics, 143A, 1338-1341. http://dx.doi.org/10.1002/ajmg.a.31774

[37] Teufel, A., Weinmann, A., Kahaly, G.J., Centner, C., Piendl, A., Wörns, M., Lohse, A.W., Galle, P.R. and Kanzler, S. (2010) Concurrent Autoimmune Diseases in Patients with Autoimmune Hepatitis. Journal of Clinical Gastroente-rology, 44, 208-213. http://dx.doi.org/10.1097/MCG.0b013e3181c74e0d

[38] Chen, J.J. and Allen, R.C. (2014) Ocular Sarcoidosis and Immune Thrombocytopenia: A Rare Association. Orbit, 33, 62-64. http://dx.doi.org/10.3109/01676830.2013.842255

[39] Hofbauer, L.C., Spitzweg, C. and Schmauss, S. (1997) Graves Disease Associated with Autoimmune Thrombocytopenic Purpura. Archives of Internal Medicine, 157, 1033-1036. http://dx.doi.org/10.1001/archinte.1997.00440300155015

[40] Wiesneth, M., Pflieger, H., Frickhofen, N. and Heimpel, H. (1985) Idiopathic Combined Immunocytopenia. British Journal of Haematology, 61, 339-348. http://dx.doi.org/10.1111/j.1365-2141.1985.tb02834.x

[41] Evans, R.S. and Duane, R.T. (1949) Acquired Hemolytic Anemia: Relation of Erythrocyte Antibody to Activity of the Disease, Significance of Thrombocytopenia and Leukopenia. Blood, 4, 1196-1213.

[42] Pratt, E.L., Tarantino, M.D., Wagner, D., Hirsch Pescovitz, O., Bowyer, S. and Shapiro, A.D. (2005) Prevalence of Elevated Antithyroid Antibodies and Antinuclear Antibodies in Children with Immune Thrombocytopenic Purpura. American Journal of Hematology, 79, 175-179. http://dx.doi.org/10.1002/ajh.20299

[43] Altintas, A., Ozel, A., Okur, N., Okur, N., Cil, T., Pasa, S. and Ayyildiz, O. (2007) Prevalence and Clinical Significance of Elevated Antinuclear Antibody Test in Children and Adult Patients with Idiopathic Thrombocytopenic Purpura. Journal of Thrombosis and Thrombolysis, 24, 163-168. http://dx.doi.org/10.1007/s11239-007-0031-y

[44] Kurata, Y., Miyagawa, S., Kosugi, S., Kashiwagi, H., Honda, S., Mizutani, H., Tomiyama, Y., Kanayama, Y. and Matsuzawa, Y. (1994) High-Titer Antinuclear Antibodies, Anti-SSA/Ro Antibodies and Anti-Nuclear RNP Antibodies in Patients with Idiopathic Thrombocytopenic Purpura. Thrombosis and Haemostasis, 71, 184-187.

[45] Demir, C., Esen, R., Atmaca, M. and Efe, S. (2010) Prevalence of Autoantibodies Related to Some Autoimmune Disorders in Patients with Chronic Idiopathic Thrombocytopenic Purpura. Clinical and Applied Thrombosis/Hemostasis, 17, E114-E118. http://dx.doi.org/10.1177/1076029610387588 
[46] Bay, A., Coskun, E., Leblebisatan, G., Karaoglu, O., Keskin, M., Yavuz, S., Yilmaz, F., Dai, A. and Coskun, M.Y. (2013) Prevalence and Clinical Significance of Antithyroid Antibodies in Children with Immune Thrombocytopenic Purpura. Pediatric Hematology and Oncology, 30, 698-704. http://dx.doi.org/10.3109/08880018.2012.756564

[47] Dash, S., Marwaha, R.K. and Mohanty, S. (2004) Lupus Anticoagulant in Immune Thrombocytopenic Purpura. The Indian Journal of Pediatrics, 71, 505-507. http://dx.doi.org/10.1007/BF02724290

[48] Javierre, B.M., Hernando, H. and Ballestar, E. (2011) Environmental Triggers and Epigenetic Deregulation in Autoimmune Disease. Discovery Medicine, 12, 535-545.

[49] Li, H., Xuan, M. and Yang, R. (2013) DNA Methylation and Primary Immune Thrombocytopenia. Seminars in Hematology, 50, S116-S126. http://dx.doi.org/10.1053/j.seminhematol.2013.03.022

[50] Rose, N.R. (1997) Autoimmune Diseases: Tracing the Shared Threads. Hospital Practice, 32, 147-154. http://dx.doi.org/10.1080/21548331.1997.11443469

Submit or recommend next manuscript to OALib Journal and we will provide best service for you:

- Publication frequency: Monthly

- 9 subject areas of science, technology and medicine

- Fair and rigorous peer-review system

- Fast publication process

- Article promotion in various social networking sites (LinkedIn, Facebook, Twitter, etc.)

- Maximum dissemination of your research work

Submit Your Paper Online: Click Here to Submit

Contact Us: $\underline{\text { service@oalib.com }}$ 